\title{
Chaotic Oscillator Based on Mathematical Model of Multiple-Valued Memory Cell
}

\author{
Jiri Petrzela \\ Department of Radio Electronics \\ Brno University of Technology \\ Brno, Czech Republic \\ petrzelj@feec.vutbr.cz
}

\begin{abstract}
This paper describes development of analog chaotic oscillator based on mathematical model of static multiple-valued memory system. Underlying dynamics is covered by set of three ordinary differential equations without driving force and stochastic processes. Existence of chaos is proved both numerically by calculation of the largest Lyapunov exponent (LLE) and experimentally by real laboratory experiments; these can be considered as evidence of the robustness and structural stability of the observed strange attractors. Even though analyzed dynamical system is topologically conjugated to famous Chua's oscillator (proved in paper) discovered circuitry can be considered as novel chaotic oscillator.
\end{abstract}

Keywords-analog oscillator; chaos; linear topological conjugacy; Lyapunov exponents; nonlinear dynamics; static memory; strange attractors

\section{INTRODUCTION}

Multiple-valued static system (MVSM) system has been already utilized in various practical applications [1]-[3] and studied from different perspectives [4]-[7] for decades. In these papers, autonomous deterministic dynamical system with three degrees of freedom has been addressed. In the simplest case (see Fig. 1), two parallel resonant diodes (RTD) are connected in series. For high-frequency applications, RTD can be modeled by the equivalent circuit composed by piecewise linear (PWL) two-terminal element [8] with N-type amperevoltage characteristics, inductor and resistor connected in series (both represent properties of RTD leads) and parallel capacitor. However, chaos was not reported as possible solution. Instead of this, numerical analysis led authors to the conclusion that only fixed points and limit cycles are observable within MVSM dynamics. Upcoming sections invalidate this statement; although numerical values of the circuit parameters leading to chaos are far away from a common operational regime of MVSM.

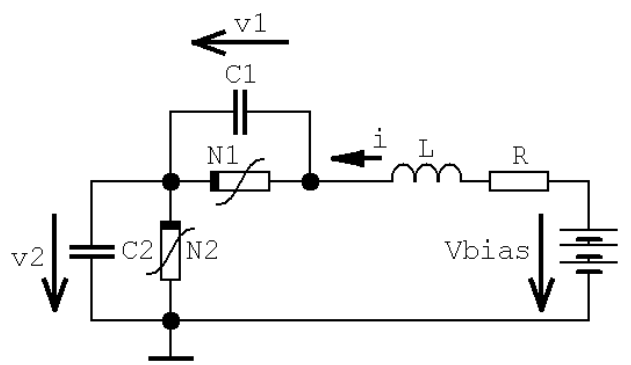

Figure 1. Fundamental network capable to model dynamical behavior associated with MVSM.

\section{MATHEMATICAL DESCRIPTION OF MEMORY}

Circuit that represents MVSM system provided in Fig. 1 can be described by a following set of ordinary differential equations

$$
\begin{aligned}
& C_{1} \frac{d v_{1}}{d t}=i-f\left(v_{1}\right) \\
& C_{2} \frac{d v_{2}}{d t}=i-\frac{v_{2}}{R_{e}} \\
& L \frac{d i}{d t}=V_{\text {bias }}-v_{1}-v_{2}-R \cdot i
\end{aligned}
$$

where $V_{\text {bias }}$ is a biasing voltage for both RTD, second RTD is linearized and can be replaced by resistor $R_{e}$. Scalar PWL approximation of ampere-voltage curve of first RTD can be expressed as

$$
f(x)=m_{0} \cdot x+\sum_{n=1}^{2} m_{n}\left|x-V_{n}\right|+m_{3},
$$

has two breakpoints $V_{1,2}$ while slopes in the individual segments of the vector field can be calculated using $g_{\text {lefi }}=m_{0}-m_{1}-m_{2}, g_{\text {inner }}=m_{0}+m_{1}-m_{2}$ and $g_{\text {right }}=m_{0}+m_{1}+m_{2}$ respectively. These slopes affect global behavior since these contributes to eigenvalues because characteristic polynomial has following form

$$
\begin{aligned}
& \lambda^{3}+\left(\frac{R}{L}+\frac{g}{C_{1}}+\frac{1}{C_{2} R_{e}}\right) \lambda^{2}+ \\
& +\left[\frac{1}{C_{1} L}(1+g \cdot R)+\frac{1}{C_{2} L}\left(1+\frac{R}{R_{e}}\right)+\frac{g}{C_{1} C_{2} R_{e}}\right] \lambda+ \\
& +\frac{1}{C_{1} C_{2} L}\left(g+\frac{1}{R_{e}}+g \frac{R}{R_{e}}\right)=0
\end{aligned}
$$

where $g$ is $g_{\text {left }}, g_{\text {inner }}$ or $g_{\text {right }}$ depending on the actual segment of vector field. Let's assume all accumulation elements are positive and there is just one fixed point per state space region. Also suppose that desired state attractor is large enough to activate all slopes of RTD such that $g_{\text {left }}>0, g_{\text {inner }}<0$ and $g_{\text {right }}>0$. These premises restrict reachability of the eigenvalues and a potential chance to obtain geometry of a vector field suitable for evolution of chaos. Despite of this, a mentioned space composed by MVSM parameters represents standard operation regime of MVSM. In further text, violation of this space is admitted. 
Pair of the differently-shaped strange attractors has been localized within dynamics of MVSM system (1). First one can be observed for the following normalized values $C_{1}=47 \mathrm{mF}, C_{2}=4.64 \mathrm{~F}, L=425 \mathrm{mH}, R=2.42 \Omega$, $R_{e}=240 \mathrm{~m} \Omega, g_{\text {lefi }}=g_{\text {right }}=396 \mathrm{mS}, g_{\text {inner }}=-312 \mathrm{mS}$. These values lead to the eigenvalues in the outer regions of a state space $\lambda_{1,2}=0.207 \pm \mathrm{j} 1.013, \lambda_{3}=-2.246$ and in inner segment of a vector field $\lambda_{1,2}=0.333 \pm \mathrm{j} 3.496, \lambda_{3}=0.62$. Thus, local geometry is formed by the saddle-focusses with stability index 1 and 2 respectively.

Second type of a chaotic attractor can be observed by adopting normalized values $C_{1}=2.32 \mathrm{~F}, C_{2}=-1.545 \mathrm{~F}$, $L=50 \mathrm{mH}, R=-48 \mathrm{~m} \Omega, R_{e}=20 \Omega$ and symmetrical outer slopes $g_{\text {lefi }}=g_{\text {right }}=-3 \mathrm{~S}$ with $g_{\text {inner }}=6 \mathrm{~S}$. Eigenvalues are $\lambda_{1,2}=0.94 \pm \mathrm{j} 2.536, \lambda_{3}=2.245$ and in the inner segment of a vector field we get $\lambda_{1,2}=0.227 \pm \mathrm{j} 2.905, \lambda_{3}=-3.967$. Local geometry is a composition of the saddle-focus equilibrium with stability index 2 and 1 respectively.

Existence of both chaotic attractors was proved by numerical analysis, namely using Mathcad and buildin fourth-order Runge-Kutta method with a final time $10^{4}$ and fixed time step 0.01 . Corresponding results are shown in Fig. 2 and Fig. 3. Common notation for these attractors is a double-scroll [9], [10] and dual doublescroll [11].

If one RTD is linearized and the remaining one is approximated by a PWL function with three segments studied dynamical system is topologically conjugated to the well-known Chua's oscillator. It means that MVSM belongs into the so-called dynamical systems of class C; nice description can be found in [12]. Thus, MVSM can model rich scale of associated dynamical motion including chaos. Complete transformation $\mathbf{T}$ between two members of class $\mathrm{C}$ can be established through partial transformations $\mathbf{K}$ to the normal form. In detail, a linear transformation of the coordinates that transform analyzed MVSM into normal form is

$$
\mathbf{K}=\left(\begin{array}{ccc}
1 & 0 & 0 \\
-\frac{g_{1}}{C_{1}} & 0 & \frac{1}{C_{1}} \\
\left(\frac{g_{1}}{C_{1}}\right)^{2}-\frac{1}{C_{1} L} & -\frac{1}{C_{1} L} & -\frac{g_{1}}{C_{1}^{2}}-\frac{R}{C_{1} L}
\end{array}\right),
$$

and derived dynamical system in normal form is

$$
\begin{aligned}
& \left(\begin{array}{c}
\dot{x} \\
\dot{y} \\
\dot{z}
\end{array}\right)=\left(\begin{array}{ccc}
0 & 1 & 0 \\
0 & 0 & 1 \\
-\frac{g_{\text {outer }}\left(R+R_{e}\right)+1}{\beta} & -\frac{\alpha}{\beta} & -\delta
\end{array}\right) \cdot\left(\begin{array}{l}
x \\
y \\
z
\end{array}\right)+ \\
& +\frac{1}{C_{1}}\left(\begin{array}{c}
\phi \\
-g_{\text {outer }} \phi / C_{1} \\
\phi \cdot \chi
\end{array}\right) \frac{1}{2}(|x+1|-|x-1|)+ \\
& +\left(0 \frac{V_{\text {bias }}}{C_{1} L}-\frac{V_{\text {bias }}\left(g_{\text {outer }} / C_{1}+R / L\right) / C_{1}}{L}\right)^{T}
\end{aligned}
$$

Remaining parameters are

$$
\begin{aligned}
& \alpha=C_{1}\left(R+R_{e}\right)+C_{2} R_{e}\left(1+g_{\text {outer }} R\right)+g_{\text {outer }} L \\
& \chi=\frac{1}{C_{1}}-\left(\frac{g_{\text {outer }}}{C_{1}}\right)^{2} .
\end{aligned}
$$

Observed strange attractors after a linear change of the coordinates are demonstrated in Fig. 4. These state trajectories serve as the reference orbits for upcoming comparison with experimental verification. Dynamical system in a normal form produce significantly smaller attractors; it means that smaller state space volume is required to fully cover attractor. From the viewpoint of practical realization, this is indeed advantageous since it prevents active elements to enter saturation.

In upcoming practical realization of MVSM-based chaotic oscillators a saturation-type PWL function will be considered as two-port $h(x)=(|x+1|-|x-1|)$.

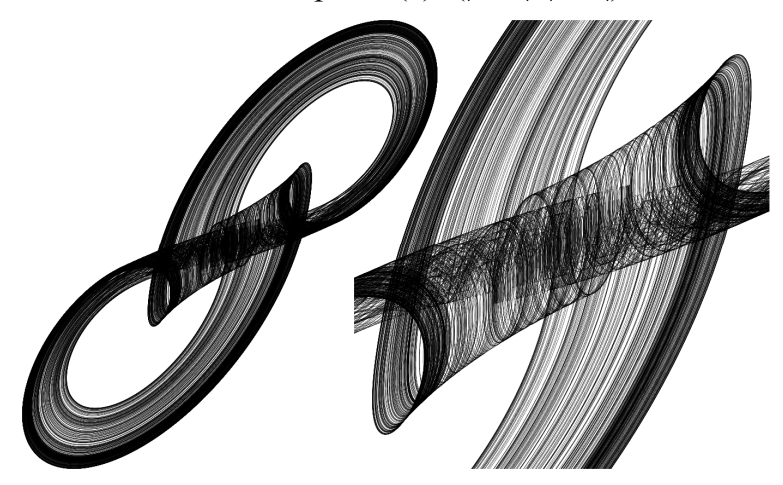

Figure 2. Three-dimensional view on strange attractor generated by first configuration of MVSMS (left picture), detailed zoom on inner segment of a vector field (right plot).

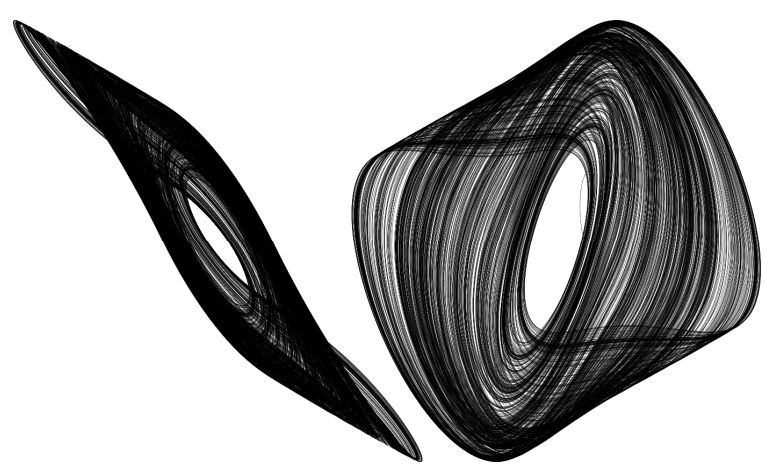

Figure 3. Different three-dimensional views on strange attractor generated by second configuration of MVSM system.

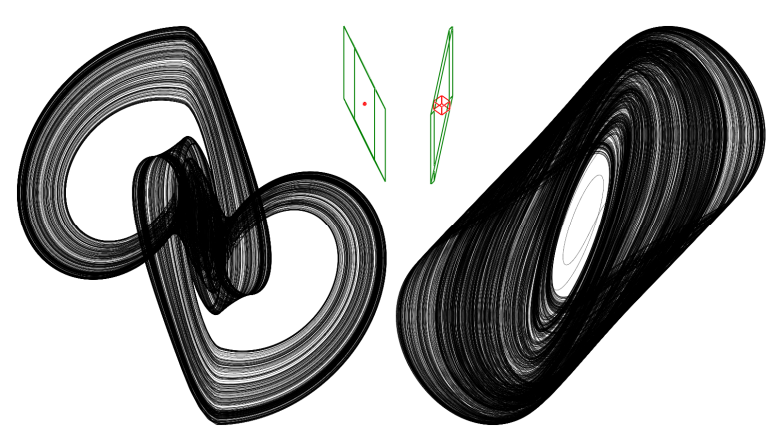

Figure 4. Strange attractors transformed into the normal form, state space deformation under transformation of coordinates $\mathbf{K}$ : original system (green), normal form (red). 


\section{CIRCUITRY REALIZATION OF CHAOTIC MVSM}

Note that few negative immittance converters must be employed to construct a chaotic MVSM having the announced parameters. However, original MVSM (1) transformed into the normal form represents a cascade connection of two-port integrators with a multi-branch linear and nonlinear feedback. Such network structure can be designed using analog computer concept [13], [14]. Two sets of the ordinary differential equations with the normalized numerical values to be realized as first and second analog electronic circuit are following

$$
\begin{aligned}
& d x / d t=y-1.76 \cdot h(x) \\
& d y / d t=z-2.07 \cdot h(x) \\
& d z / d t=-2.3 \cdot x-0.86 \cdot y+0.1 \cdot z+4.8 \cdot h(x) \\
& d x / d t=y-3.87 \cdot h(x) \\
& d y / d t=z-5.02 \cdot h(x) \\
& d z / d t=16.42 \cdot x-3.1 \cdot y+0.37 \cdot z-40 \cdot h(x)
\end{aligned}
$$

Circuitry implementations of both chaotic MVSM systems are very similar; see Fig. 5 and Fig. 6 for first and second set of parameters. Note that both networks are very similar and supplied using symmetrical $\pm 15 \mathrm{~V}$. Time constant for the chaotic circuits were chosen low $\tau=R \cdot C=10^{4} 10^{-7}=1 \mathrm{~ms}$ such that high-frequency parasitic properties of the active elements can be neglected. Summing-up couple of the currents implement PWL function: in detail, a continual contribution through resistor $R_{x}$ and portion that acts only in outer segments of a vector field, i.e. current through resistor $R_{y}$.

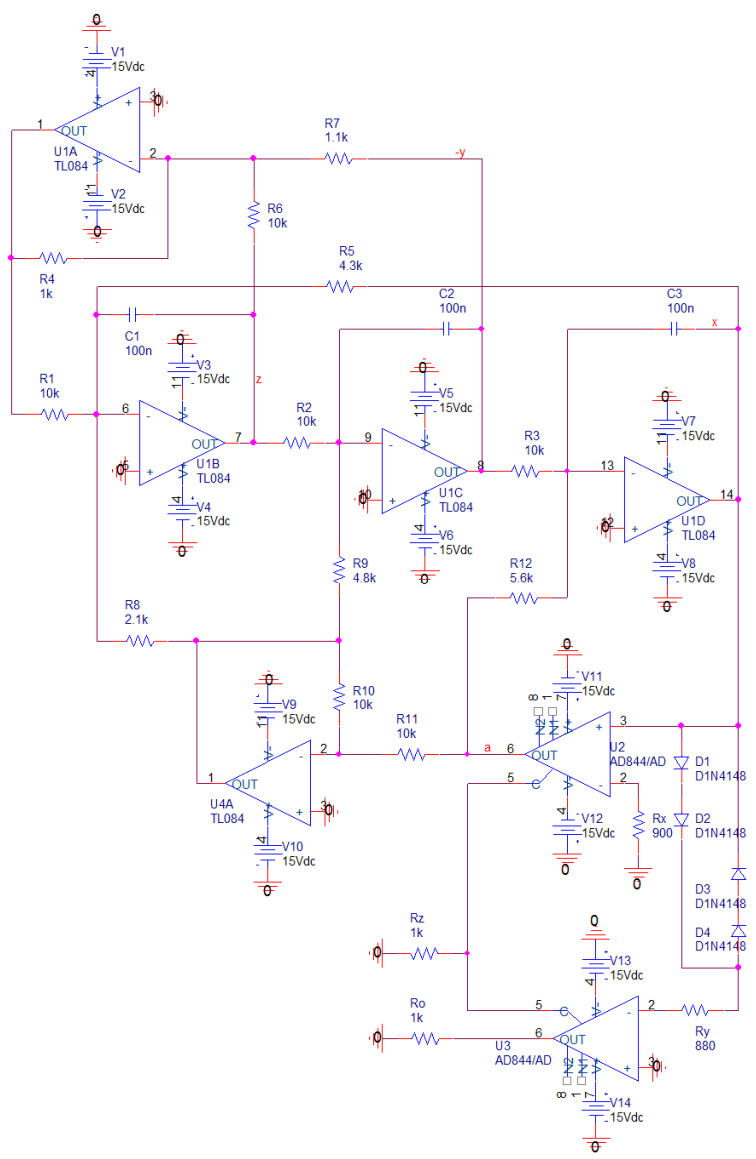

Figure 5. Chaotic oscillator ready to generate double-scroll attractor in Orcad Pspice circuit simulator; MVSM dynamical system with first set of the parameters.

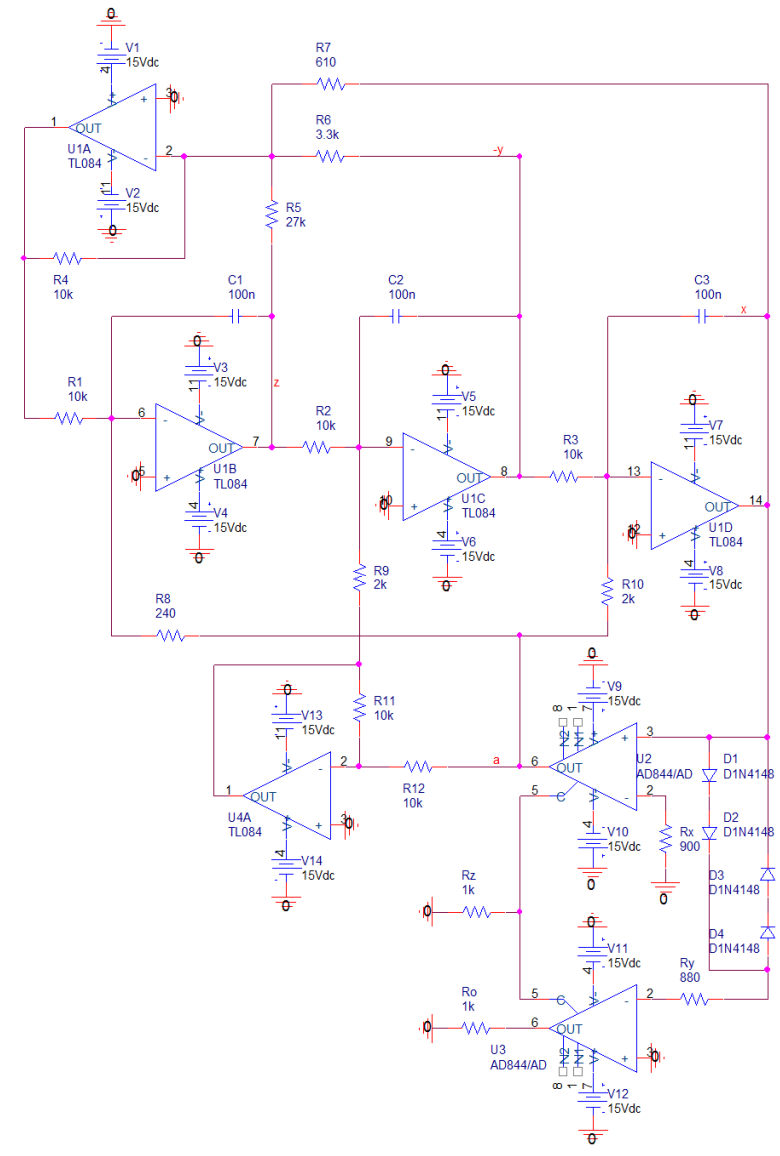

Figure 6. Chaotic oscillator ready to generate double-scroll attractor in Orcad Pspice circuit simulator; MVSM dynamical system with second set of the parameters.

State variables $\mathbf{x}=(x,-y, z)^{\mathrm{T}}$ are easily accessible as voltages at outputs of individual inverting integrators. In circuit given in Fig. 5 resistors $R_{6}, R_{8}$ and $R_{9}$ were replaced by the linear potentiometers to trace route-tochaos scenarios. In the case of second MVSM-based circuit only two resistors were assumed to be a handvariable; namely $R_{8}$ and $R_{9}$. Shape of PWL function can be visualized as voltage $V(a)$ vs $V(x)$.

\section{Simulation And Measurement Results}

It is a standard habit to verify existence of strange attractor by computer-aided simulations as well as real laboratory measurement. If second sort of outputs exist Orcad Pspice simulation results usually provide same results and can be omitted. Oscilloscope screenshots showing selected plane projections generated by first and second chaotic MVSM-based oscillator is given in Fig. 7 and Fig. 8 respectively. During experimental verification it turns out that proposed integrator-based conception of chaotic oscillator is extremely sensitive to the values of the passive circuit components which should be chosen precisely up five percent. Regions in the parameter space leading to evolution of a chaotic attractor are narrow and surrounded by a fixed-point solution or large limit cycle (given by saturation levels of the active elements). Some interesting experimental observations are provided in Fig. 9. These screenshots show vector field positions and geometrical relations between individual solutions of investigated system. Good final agreement between theoretical expectations and experimental results has been confirmed. 


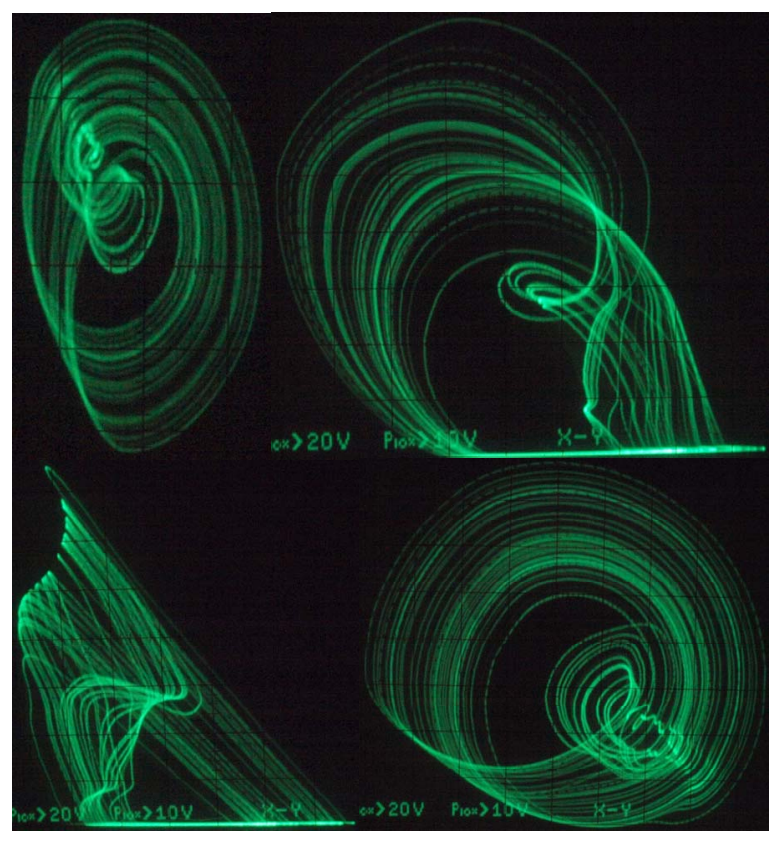

Figure 7. Strange attractor generated by first chaotic oscillator.

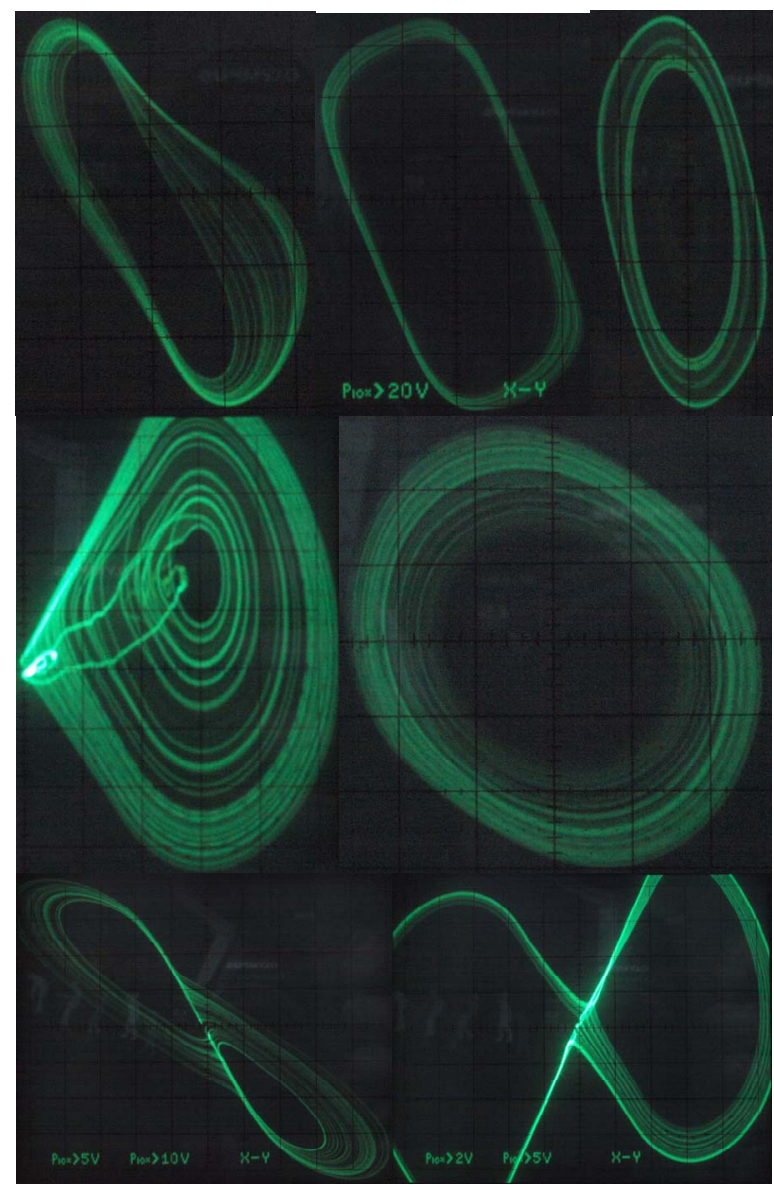

Figure 8. Strange attractor generated by second chaotic oscillator.

\section{CONClusion}

This brief paper demonstrates that standard circuit configuration of MVSM can be used as autonomous analog generator of continuous chaotic signals. Instead of a construction of negative capacitors and inductors, straightforward integrator-based synthesis was applied on the design process of the chaotic oscillators.

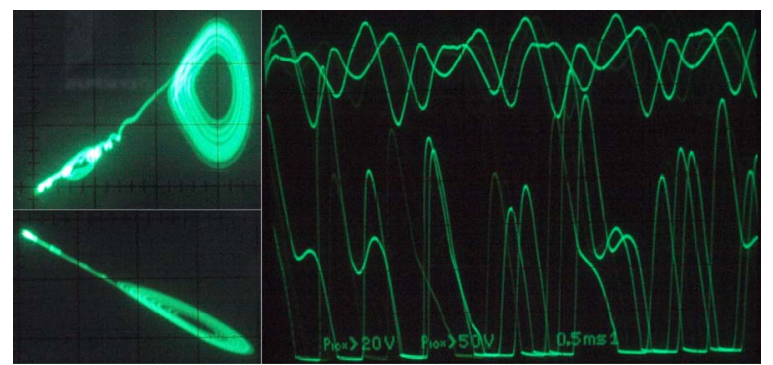

Figure 9. Transient observations: fixed point (origin) and formation of single-scroll attractor in different plane projections, chaotic waveforms $x(t)$ and $y(t)$ in time domain.

\section{ACKNOWLEDGMENT}

Research described in this paper was financed by National Sustainability Program under grant LO1401. For research, infrastructure of SIX Center was used.

\section{REFERENCES}

[1] J. T. Butler, "Multiple-valued logic," IEEE Potentials, vol. 14, pp. 11-14, 1995.

[2] H. Nan, K, Choi, "Novel ternary logic design based on CNFET," In Proceedings of International SoC Design Conference ISOCC 2010, Seoul (South Korea).

[3] J. Nunez, M. J. Avedillo, J. M. Quintana, "Evaluation of RTD-CMOS logic gates," In Proceedings of $13^{\text {th }}$ Euromicro Conference on Digital System Design: Architectures, Methods and Tools DSD 2010, Lille (France).

[4] M. Guzan, "Boundary surface of a ternary memory in the absence of limit cycles ," In Proceedings of $22^{\text {nd }}$ International Conference Radioelektronika 2012, Brno (Czech Republic).

[5] P. Galajda, M. Guzan, V. Spany, "The state space mystery with negative load in multiple-valued logic," Radioengineering, vol. 8, no. 2, pp. 2-7, 1999.

[6] M. Guzan, "Analysis of 6(4) - valued memory," Elektronika Ir Elektrotechnika, vol. 20, no. 6, pp. 89-92, 2014.

[7] V. Spany, L. Pivka, "Boundary surfaces in sequential circuits," International Journal of Circuit Theory and Applications, vol. 18, pp. 349-360, 1990.

[8] W. R. Liou, P. Roblin, "High frequency simulation of resonant tunelling diodes," IEEE Trans. on Electronic Devices, vol. 41, pp. 1098-1111, 1994.

[9] L. Chua, M. Komuro, T. Matsumoto, "The double scroll family," IEEE Transactions on Circuits and Systems, vol. 33, no. 11, pp. 1072-1118, 1986.

[10] M. Guzan, "Chua's singularities and presence of chaos," In Proceedings of $17^{\text {th }}$ International Conference on Electrical Drives and Power Electronics, High Tatras (Slovakia), 2011.

[11] T. Parker, L. Chua, "The dual double scroll equation," IEEE Transactions on Circuits and Systems, vol. 34, no. 9, pp. 1059-1073, 1987.

[12] J. Pospisil, Z. Kolka, J. Horska, J. Brzobohaty, "Simplest ODE equivalents of Chua's equations," International Journal of Bifurcations and Chaos, vol. 10, no. 1, pp. 1-23, 2000.

[13] J. Petrzela, Z. Hrubos, T. Gotthans, "Modeling deterministic chaos using electronic circuits," Radioengineering, vol. 20, no. 2, pp. 438-444, 2011.

[14] M. Itoh, "Synthesis of electronic circuits for simulating nonlinear dynamics," International Journal of Bifurcation and Chaos, vol. 11, no. 3, pp. 605-653, 2001. 